\title{
Septoplastía endoscópica
}

\section{Endoscopic septoplasty}

\author{
Rodolfo Nazar S', Natalia Cabrera S², Alfredo Naser G'.
}

\section{RESUMEN}

La septoplastía es uno de los procedimientos quirúrgicos más frecuentes en otorrinolaringología, cuya principal indicación es la presencia de desviación septal nasal significativa. Este enfoque quirúrgico ha evolucionado en forma considerable en las últimas décadas, siendo la septoplastía endoscópica la técnica más reciente. Esta técnica moderna, basada en la septoplastía convencional, ha mostrado beneficios en cuanto al procedimiento, resultados y complicaciones. El objetivo de este artículo es revisar las indicaciones, técnica, ventajas y principales resultados de la septoplastía endoscópica.

Palabras clave: Obstrucción nasal, septoplastía, espolón septal, sinequias.

\begin{abstract}
Septoplasty is one of the most common surgical procedures in otolaryngology, whose main indication is the presence of significant nasal septal deviation. This surgical approach has evolved considerably in recent decades, and endoscopic septoplasty is the most recent technique. This modern technique based on conventional septoplasty has shown several benefits in terms of procedure, results and complications. The aim of this article is to review the indications, techniques, advantages and main results of endoscopic septoplasty.
\end{abstract}

Key words: Nasal obstruction, septoplasty, septal spur, synechiae.

\footnotetext{
${ }^{1}$ Médico Otorrinolaringólogo, Departamento de Otorrinolaringología, Hospital Clínico Universidad de Chile.

${ }^{2}$ Interna de Medicina, Universidad de Chile.
} 


\section{INTRODUCCIÓN}

La obstrucción nasal es el síntoma más común y el principal motivo de consulta en la práctica rinológica ${ }^{1}$. Dentro de las principales causas de este síntoma (Tabla 1), destaca la septodesviación, la cual produce obstrucción al obliterar más del $50 \%$ de la válvula nasal anterior ${ }^{2}$.

Es importante destacar que la septodesviación también es en algunas ocasiones causa de epistaxis, sinusitis, SAOS y cefalea, por puntos de contacto con estructuras de la pared nasal lateral' ${ }^{1}$. Se ha encontrado asociación clínica de la apnea obstructiva del sueño con la septodesviación, sin encontrarse correlación lineal entre el grado de obstrucción y la severidad del SAOS, por lo que la corrección del defecto no corrige la patología del sueño ${ }^{5}$.

Se debe realizar una evaluación médica completa antes de plantear el tratamiento quirúrgico de la septodesviación mediante septoplastía, por lo que se debe incluir la evaluación de los deseos del paciente, principalmente en relación a la realización de rinoplastia, y conocer de manera precisa las alteraciones anatómicas y funcionales nasales, mediante rinoscopia anterior, endoscopía nasal, rinometría acústica, rinomanometría y tomografía computarizada de cavidades paranasales ${ }^{6,7}$.

En cuanto a la rinometría acústica y rinomanometría, se ha encontrado una débil correlación entre las mediciones subjetivas (expresión de la sensación de obstrucción nasal) y objetivas (rinometría acústica y rinomanometría) de la obs- trucción nasal, por lo que la decisión quirúrgica debe basarse principalmente en los hallazgos en la rinoscopía, tomografía computarizada y la endoscopía nasal, usando los métodos objetivos como apoyo diagnóstico cuando se pesquisa obstrucción mecánica ${ }^{8}$.

La septoplastía es la técnica quirúrgica que tiene como finalidad la corrección de las deformidades del tabique nasal ${ }^{9}$. En Chile no se encuentran datos estadísticos publicados sobre su frecuencia, pero en Estados Unidos se estima que se realizan 340.000 septoplastías al año $0^{10}$.

Sus principales indicaciones se detallan a continuación $n^{11,12:}$

- Obstrucción nasal sintomática.

- Deformidad de la nariz.

- Acceso para cirugía endoscópica funcional de CPN o dacriocistorinotomía.

- Acceso para cirugía transesfenoidal.

- Corrección de alteraciones anatómicas que predisponen a sinusitis crónica o recurrente.

- Mejoría de acceso postoperatorio para futuros procedimientos.

- Alivio de cefalea por puntos de contacto.

\section{RESEÑA HISTÓRICA}

Las primeras descripciones de este procedimiento fueron encontradas en papiros egipcios, principalmente en relación con el tratamiento de fracturas de septum nasal, donde se introducían dos tapones de lino recubierto con grasa dentro de

Tabla 1. Causas de obstrucción nasal

\begin{tabular}{|lll|}
\hline Clasificación & Tipos & Ejemplos \\
\hline Mecánicas & Congénitas & Encefalocele, teratoma, glioma, atresia coanal \\
& Anatómicas & Septodesviación, colapso válvula nasal, sinequias, hipertrofia cornetes \\
& Tumorales & Pólipos nasales, neoplasias benignas y malignas \\
& Traumáticas & Cuerpo extraño, perforación septal \\
& latrogénicas & Rinitis atrófica, resección excesiva en osteotomías \\
\hline \multirow{2}{*}{ Funcionales } & Alérgicas & Rinitis alérgica \\
& Endocrinas & Embarazo, hipotiroidismo, insuficiencia adrenal, mentruación \\
& Infecciones & Rinosinusitis aguda y crónica, absceso septal \\
& Fármacos & Rinitis medicamentosa, reacción adversa a fármacos \\
& Reumatológicas & Granulomatosis de Wegener \\
\hline
\end{tabular}

Adaptado de referencias 3 y 4 . 
cada fosa nasal, y se situaban rodillos rígidos de lino externamente para fijar la fractura ${ }^{1,7}$.

En el siglo XIX, el procedimiento más común fue la operación de Bosworth, la cual consistía en amputar la desviación junto con la mucosa del lado convexo del septum, lo cual producía una alta tasa de perforaciones septales ${ }^{1}$.

A inicios del siglo XX, Killian y Freer describieron la técnica de la resección submucosa, la cual es aún utilizada y es la base de la septoplastía moderna. Consiste en elevar el submucopericondrio y posteriormente resecar el cartílago septal, dejando la mucosa intacta ${ }^{1,4,5}$.

Cottle, a mediados del siglo XX, describió la técnica convencional tal como se conoce actualmente, realizando la incisión hemitransfixiante en la mucosa con la posterior confección de túneles superiores e inferiores para exponer el septum, siempre respetando un bastidor en forma de "L" que soporta la estructura nasal ${ }^{1,4}$.

La aplicación de técnicas endoscópicas para corrección de septodesviaciones fue descrita al mismo tiempo por Stammberger y por Lanza y cols. en 1991. En 1993, Lanza y cols., describieron el abordaje endoscópico para el tratamiento del espolón septal5,12-14.

\section{CONSIDERACIONES ANATÓMICAS}

El septum nasal está conformado por un componente membranoso por anterior (de tejido fibroadiposo), luego un componente cartilaginoso (cartílago cuadrangular), y por posterior el componente óseo (lámina perpendicular del etmoides, cresta nasal del hueso palatino y del maxilar y el vómer). Es bordeado antero-superiormente por los huesos nasales, por inferior por los cartílagos laterales, por superior por la placa cribiforme y el proceso nasal del frontal, y postero-superiormente por el esfenoides. Las superficies están cubiertas por mucopericondrio y mucoperiostio $0^{1,4,12}$.

La irrigación proviene de las arterias etmoidales posterior y anterior, arteria esfenopalatina y arteria labial superior. La inervación es de origen trigeminal, y se distribuye a través del nervio nasopalatino que inerva la mitad postero-inferior, y los nervios etmoidales anteriores que inervan la mitad antero-superior. Es importante destacar el aporte de la inervación sensorial mediante el nervio olfatorio ${ }^{1}$.

\section{GENERALIDADES DE LA SEPTOPLASTÍA CONVENCIONAL}

El término septoplastía puede implicar una operación simple para marcar el tabique, eliminar una parte del tabique, o realizar una resección submucosa septal para ser utilizada en una rinoplastía ${ }^{7}$, aunque generalmente el procedimiento implica identificar el área específica de desviación septal y resecar esa área específica, removiendo la menor cantidad de cartílago ${ }^{1}$.

La septoplastía convencional es un procedimiento realizado mediante visualización directa de la cavidad nasal usando luz frontal y espéculo nasal, por lo que la visión del campo es limitada, en especial en la visualización del tercio inferior y posterior del septum. En determinadas ocasiones, resulta difícil determinar la relación entre septum y pared nasal lateral, y también dificulta la completa evaluación del septum posterior. Una de sus principales ventajas es que requiere instrumentación simple, y además es un procedimiento rápido cuando es realizado por cirujanos experimentados ${ }^{12}$.

\section{SEPTOPLASTÍA ENDOSCÓPICA}

El objetivo inicial de la septoplastía endoscópica es reparar deformidades septales puntuales que dificultan el abordaje al meato medio y los senos paranasales, durante la Cirugía Endoscópica Nasosinusal (CENS), lo cual permite mejorar la exposición y acceso al campo operatorio ${ }^{14}$.

Las principales indicaciones de esta técnica son ${ }^{14}$ :

- Resección de espolón o corrección de desviación septal limitada, que comprometa el acceso endoscópico al cornete y meato medio.

- Desviaciones septales postero superiores que obstruyan el ostium del seno esfenoidal.

- Septoplastía primaria como tratamiento de la obstrucción nasal.

- Cirugía revisional.

- Técnica complementaria en Rinoplastía (minimizando área resecada para mejorar resultado estético y funcional). 
- Corrección de espolón septal como responsable de cefalea rinogénica.

- Resección de desvíos septales posteriores que dificulten el tratamiento quirúrgico de epistaxis.

- Alivio de cefalea por puntos de contacto.

Existen varias ventajas de esta técnica por sobre el procedimiento convencional, destacando la mejor visualización del campo, ya que permite mayor magnificación, iluminación y exposición de la cavidad nasal, sobre todo en el óptimo reconocimiento de los planos de tejidos, siendo un procedimiento de mayor seguridad en procedimientos quirúrgicos en pacientes pediátricos ${ }^{14,15}$. Esta mejor visión de campo minimiza las laceraciones en la mucosa debido a la disección limitada, lo cual permite el reconocimiento precoz de estas lesiones y disminuye el riesgo de perforación permanente ${ }^{11}$.

Permite el abordaje puntual de desviaciones septales localizadas, espolones septales, perforaciones y sitios de contacto, lo cual permite realizar un procedimiento más conservador ${ }^{14}$.

Otro punto a destacar, es el menor tiempo quirúrgico al realizar una septoplastía endoscópica limitada, y también cuando se requiere una septoplastía previo a una cirugía endoscópica funcional, ya que ambos procedimientos se realizan vía endoscópica, por lo que se ahorra el tiempo de transición entre instrumental quirúrgico ${ }^{11,15}$.

Al realizar septoplastías de revisión, este método facilita y reduce el riesgo de perforación, ya que la incisión se realiza inmediatamente anterior al área de desviación septal residual, sin necesidad de elevar la mucosa adherida por la disección previa $^{11,12}$

Por último, el utilizar el método endoscópico resulta una mejor herramienta para docencia y médico-legal, ya que se permite la visualización en pantalla del procedimiento que se está realizando $0^{11,14}$.

\section{INSTRUMENTACIÓN}

La instrumentación utilizada en la septoplastía endoscópica es básicamente la misma que se utiliza en la septoplastía convencional, destacando el uso del elevador aspirador Freer, que permite elevar colgajos de mucosa, de forma no-traumática, mientras aspira. También se utiliza la fresa microdebridador, que permite la resección de cartílago, fórceps JansenMiddleton (resección fragmentos óseos remanentes) y elevador de Cottle $e^{1,11,13}$.

\section{TÉCNICA QUIRÚRGICA DE LA SEPTOPLASTÍA ENDOSCÓPICA}

La mayoría de las descripciones de la técnica en la literatura son similares entre sí, a continuación se detallará la técnica descrita por Sautter y cols ${ }^{12}$.

La septoplastía endoscópica se inicia con la aplicación de epinefrina u oximetazolina para descongestionar la mucosa, así se procede a examinar la cavidad nasal con endoscopio 0 .

Se inyecta lidocaína al 1\% con adrenalina (1:100.000) a ambos lados del septum en el plano subpericóndrico. Se procede a realizar la incisión de Killian (en el borde caudal septal) en el lado derecho del septum, caudal a la unión mucocutánea. Luego se crea un plano submucopericóndrico, con el elevador de Cottle, a lo largo del lado izquierdo del septum, y se diseca hacia posterior con elevador Freer (Figura 1).

Se marca y se secciona el cartílago septal, dejando al menos $1 \mathrm{~cm}$ de septum hacia caudal y hacia dorsal, para mantener el soporte de la punta nasal. Luego se realiza plano submucopericóndrico en el lado opuesto al septum. Al separar cartílago y hueso de la mucosa, se utiliza el fórceps Jansen-

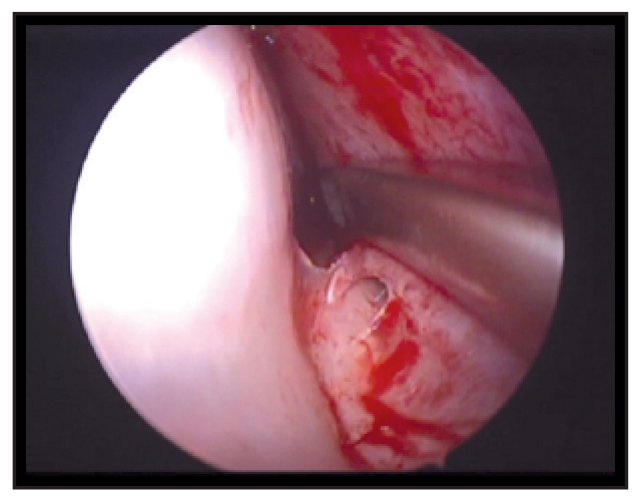

Figura 1. Disección incompleta del plano submucopericóndrico izquierdo con elevador de Cottle. 
Middleton para seccionar el septum de anterior a posterior (Figura 2).

Luego se aproximan los colgajos de mucosa y la incisión de Killian se sutura con Vicryl o Catgut (4-0). Se ha evaluado el uso de otros métodos de aproximación, como los corchetes, sin demostrarse diferencia en cuanto a comodidad ni a resistencia de flujo nasal, con respecto a la sutura ${ }^{16}$.

Finalmente, se realiza taponamiento nasal, si se requiere. En cuanto a este último punto existe evidencia limitada sobre su uso para prevención de complicaciones, tales como epistaxis, hematoma septal, sinequias y desviación septal posquirúrgica. Incluso se ha demostrado que aumenta el dolor posoperatorio, e incluso el shock tóxico ${ }^{17}$. Se han propuesto nuevas técnicas de sutura, tales como la transeptal, que han mostrado resultados equivalentes al uso de taponamiento en cuanto a epistaxis, sinequias, hematoma septal e infección local ${ }^{18}$.

\section{CONSIDERACIONES ESPECIALES}

Existen algunas situaciones en las cuales resulta especialmente útil la realización de septoplastía endoscópica, dentro de éstas se encuentra la septoplastía dirigida, indicada para el tratamiento de espolones septales aislados. En este caso la septoplastía endoscópica permite realizar una disección limitada mediante una incisión horizontal en el ápex del espolón, con posterior resección de éste con pinzas o microdebridador y cierre de colgajos de mucosa (Figura 3). Este procedimiento permite una rápida recuperación ${ }^{11-13}$.

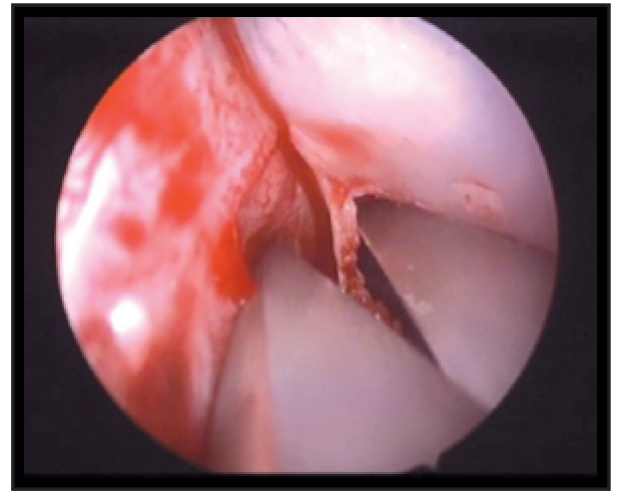

Figura 2. Sección del tabique nasal, con fórceps JansenMiddleton, para resecar espolón septal.

Otra consideración especial es el manejo de perforaciones septales, donde se realiza una incisión mucosa posterior a la perforación, disminuyendo el riesgo de aumentar su tamaño e intentando mejorar el resultado quirúrgico ${ }^{12}$.

En las septoplastías de revisión, este método permite la óptima visualización de planos tisulares alterados, minimizando el riesgo de perforación. Debido a la precisa observación se puede verificar y retomar el plano errado en algún momento ${ }^{12}$.

En septodesviaciones posteriores, la septoplastía endoscópica permite una disección posterior aislada y limitada, evitando suturas y riesgo de perforación ${ }^{11}$.

Finalmente, al terminar la intervención la visión con endoscopía permite verificar la permeabilidad de las fosas nasales, evitando revisiones a ciegas o con espéculo nasal.

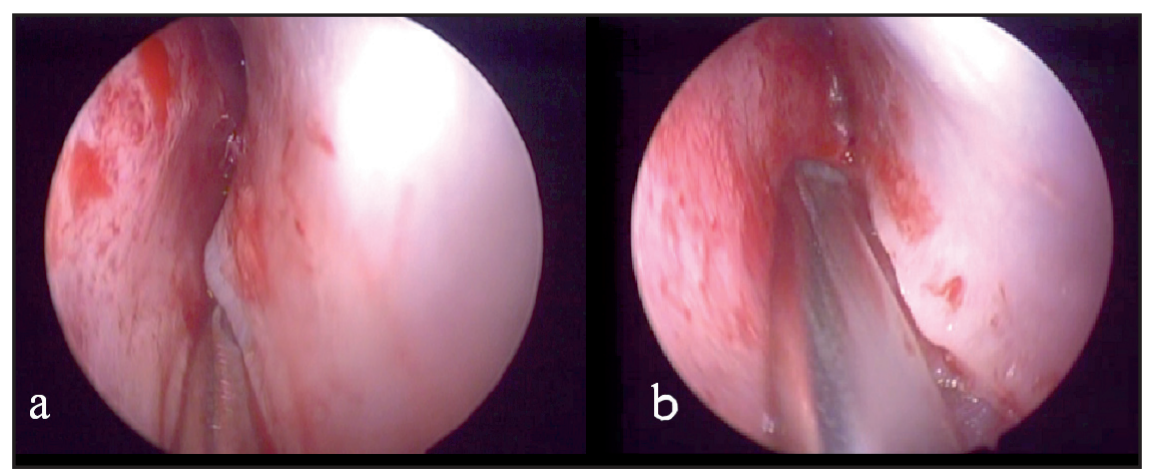

Figura 3. Disección de espolón septal derecho, bajo visión con endoscopio de 0 grados. Figura a) previo a la resección y figura b) posterior a ésta. 


\section{RESULTADOS CLÍNICOS DE LA SEPTOPLASTÍA ENDOSCÓPICA}

Los resultados clínicos de la septoplastía endoscópica son comparables a los de la técnica convencional, mostrando resolución significativa de la obstrucción nasal en el 70\%-80\% de los pacientes, en los distintos estudios ${ }^{11}$.

Algunos estudios comparativos con la técnica convencional, han encontrado que la septoplastía endoscópica es más efectiva en tratar síntomas como la obstrucción nasal y la cefalea ${ }^{19,20}$.

En cuanto a los síntomas en el periodo posoperatorio se ha observado una menor tasa de dolor, cefalea, edema facial y epífora ${ }^{21}$. También se ha observado menor tasa de formación de sinequias en los pacientes tratados con la técnica endoscópica ${ }^{21,22}$. En términos de la estadía hospitalaria, no se han encontrado diferencias estadísticamente significativas en el número de días de hospitalización ${ }^{23}$.

\section{COMPLICACIONES}

En las distintas series se han observado las mismas complicaciones de la septoplastía convencional, además éstas son infrecuentes y leves, con una tasa general de 2\%-5\% en la septoplastía endoscópica ${ }^{21}$. A continuación se enumeran las complicaciones más frecuentes ${ }^{11,12}$ :

- Perforación septal (0,9\%-3,4\%)

- Hematoma septal $(0,9 \%)$

- Dolor dental transitorio e hiperestesia $(4,3 \%)$

- Sinequias (2,6\%-4,5\%)

- Epistaxis (0,9\%)

- Septodesviación persistente $(0,9 \%)$

Se han descrito complicaciones mayores en la septoplastía convencional, pero no en la endoscópica, entre las cuales destaca la fístula de LCR, trombosis del seno cavernoso, meningitis, hemorragia subaracnoidea, absceso cerebral, enfisema periorbitario y ceguera ${ }^{11}$. El principal factor para prevenir las complicaciones es lograr una visualización óptima, en lo cual la septoplastía endoscópica tiene ventajas evidentes ${ }^{9}$.

\section{CONTRAINDICACIONES Y LIMITACIONES}

No existen contraindicaciones absolutas para la realización de septoplastía endoscópica, pero podría considerarse como contraindicación relativa cuando existe desviación del marco caudal septal y desviaciones anteriores, ya que no se obtiene una adecuada visualización endoscópica y resulta más útil el uso de luz frontal tradicional ${ }^{11,14}$. En cuanto a sus limitaciones, la principal es la pérdida de la visión binocular y la frecuente necesidad de limpieza de la punta de la óptica, especialmente cuando existe un sangramiento mayor ${ }^{14}$. Otro punto considerable, es la necesidad de contar con instrumental quirúrgico óptimo, lo cual es una importante limitación en nuestro país, ya que numerosos centros de salud no cuentan con los recursos necesarios para la realización de la técnica endoscópica.

\section{CONCLUSIONES}

La obstrucción nasal es un síntoma frecuente en otorrinolaringología, siendo la septodesviación una de las principales causas. La septoplastía es una de las cirugías rinológicas más realizadas, por lo que su variedad endoscópica, menos invasiva y con una visualización óptima, resulta una alternativa atractiva. Diversos estudios describen varias ventajas de la técnica endoscópica por sobre la convencional, siendo sus complicaciones similares a esta última.

\section{BIBLIOGRAFÍA}

1. Fettman N, Sanford T, Sindwani R. Surgical management of the deviated septum: Techinques in septoplasty. Otolaryngol Clin N Am 2009; 42 : 241-52.

2. D'ascanio L, Manzinı M. Quick septoplasty: Surgical technique and learning curve. Aesth Plast Surg 2009; 33: 814-8.

3. García J, Massegur H. Nasal obstruction. In: Anniko M, Bernal-Sprekelsen M, Bonkowsky V, Bradley P, lurato S, Editors, Otorhinolaryngology, Head and Neck Surgery. Berlin, Germany: Springer; 2010. p. 213214. 
4. ÖnercI TM. Chapter 2 Nose. In: Önerci TM, Diagnosis in Otorhinolaryngology. Berlin, Germany: Springer; 2009. p. 95.

5. GeORgalas $C$. The role of the nose in snoring and obstructive sleep apnoea: an update. Eur Arch Otorhinolaryngol 2011; 268: 1365-73.

6. BECKER DG. Septoplasty and turbinate surgery. Aesthetic Surg J 2003; 23: 393: 403.

7. Fattahi T, Quereshy F. Septoplasty: Thoughts and Considerations. J Oral Maxillofac Surg 2011; 69: e528-e532.

8. Tompos T, Garal T, Zemplen B. Sensation of nasal patency compared to rhinomanometric results after septoplasty. Eur Arch Otorhinolaryngol 2010; 267: 1887-91.

9. Ketcham AS, Han JK. Complications and management of septoplasty. Otolaryngol Clin North Am 2010; 43(4): 897-904.

10. Rudmik L, Mace J, Ferguson BJ, Smith T. Concurrent septoplasty during endoscopic sinus surgery for chronic rhinosinusitis: Does it confound outcomes assessment? Laryngoscope 2011; 121(12): 2679-83.

11. Getz A, Hwang P. Endoscopic septoplasty. Curr Opin Otolaryngol Head Neck Surg 2008; 16: 26-31.

12. Sautter N, Smith T. Endoscopic septoplasty. Otolaryngol Clin N Am 2009; 42: 253-60.

13. Hwang P, Mclaughlin R, Lanza D, Kennedy D. Endoscopic septoplasty: Indications, technique and results. Otolaryngol Head Neck Surg 1999; 120: 678-82.

14. Tabasnik M. Septoplastía Endoscópica: Conferencia 8. En: Congreso Latinoamericano de Rinología y Cirugía Facial (15: 2013: Bogotá). Bogotá, 2013.
15. Tarkan 0, Soylu L, Aydogan B, Ozdemir S. Delayed cerebrospinal fluid leakage: An unusual septoplasty complication. L Oral Maxillofac Surg 2012; 70: e298-e300.

16. GÜven Y, Cingi C, Kaya E. Septal stapler use during septum surgery. Eur Arch Otorhinolaryngol 2013; 270(3): 939-43.

17. Dubin M, PLetCheR S. Postoperative packing after septoplasty: Is it necessary? Otolaryngol Clin North Am 2009; 42(2): 279-85.

18. Certal V, Silva H, Santos T, Correia A, Carvalho C. Trans-septal suturing technique in septoplasty: a systematic review and meta-analysis. Rhinology 2012; 50(3): 236-45.

19. Nayak DR, Balakrishnan MS, Murthy MS. An endoscopic approach to the deviated nasal septum-a preliminary study. J Laryngol Otol 1998; 112: 934-99.

20. Harley DH, Powitsky ES, Duncavage J. Clinical outcomes for the surgical treatment of sinonasal headache. Otolaryngol Head Neck Surg 2003; 129: 217-21.

21. Gulati S, Wadhera R, Abuja N, Garg A, Ghal A. Comparative evaluation of endoscopic with conventional septoplasty. Indian J Otolaryngol Head Neck Surg 2009; 61: 27-9.

22. Park DH, Kim TM, Han DG, Ahn KY. Endoscopic assisted correction of the deviated nose. Aesthetic Plast Surg 1998; 22(8): 190-5.

23. GuPta M, Motwanı G. Comparative study of endoscopic aided septoplasty and traditional septoplasty in posterior nasal septal deviations. Indian J Otolaryngol Head Neck Surg 2005; 57(4): 309-11. 